\title{
A versatile framework to solve the Helmholtz equation using physics-informed neural networks
}

\author{
Chao Song $^{\star}$, Tariq Alkhalifah ${ }^{\dagger}$, Umair Bin Waheed ${ }^{\ddagger}$, \\ * Formerly Department of Physical Science and Engineering, King Abdullah University of Science
} and Technology (KAUST), Thuwal, 23955-6900, Saudi Arabia, *E-mail: chao.song@kaust.edu.sa, Presently Centre for Reservoir Geophysics, Resource Geophysics Academy, Imperial College London, London SW7 2BP, United Kingdom.

$\dagger$ Department of Physical Science and Engineering, King Abdullah University of Science and Technology (KAUST) $\overrightarrow{\mathrm{e}}$

Thuwal, 23955-6900, Saudi Arabia, *E-mail: chao.song@kaust.edu.sa,

${ }^{\ddagger}$ Department of Geosciences, King Fahd University of Petroleum and Minerals (KFUPM),

Dhahran, 31261, Saudi Arabia

\section{SUMMARY}

Solving the wave equation to obtain wavefield solutions is an essential step in illuminating the subsurface using seismic imaging and waveform inversion methods. Here, we utilize a recently introduced machine-learning based framework called physicsinformed neural networks (PINNs) to solve the frequency-domain wave equation, which is also referred to as the Helmholtz equation, for isotropic and anisotropic media. Like functions, PINNs are formed by using a fully-connected neural network (AN) to provide the wavefield solution at spatial points in the domain of interest, in which the coordinates of the point form the input to the network. We train such 2 a network by back propagating the misfit in the wave equation for the output wavefield values and their derivatives for many points in the model space. Generally, 
a hyperbolic tangent activation is used with PINNs, however, we use an adaptive sinusoidal activation function to optimize the training process. Numerical results show that PINNs with adaptive sinusoidal activation functions are able to generate frequency-domain wavefield solutions that satisfy wave equations. We also show the flexibility and versatility of the proposed method for various media, including anisotropy, and for models with strong irregular topography.

Key words: Neural Networks fuzzy logic; Numerical modelling; Seismic anisotropy; Wave propagation.

\section{INTRODUCTION}

Simulating the seismic wave propagation is one of the most fundamental problems in seismic exploration. Time-domain wave equation modelling is computationally expensive for a large number of sources, and it requires a lot of memory for storing the wavefield solutions. With the same number of sources, the frequency-domain wave equation modelling is able to save the computational cost significantly (Marfurt 1984). In addition, only a limited number of frequencies are needed to apply waveform inversion for large aperture seismic surveys (Pratt 1990, 1999). By inverting the impedance matrix of the Helmholtz equation, we can obtain frequency-domain wavefield solutions, and consequently, illuminate the Earth using seismic imaging or inversion methods, such as reverse time migration (RTM) and full-waveform inversion (FWI).

The anisotropic nature of the Earth has been recognized for decades (Uhrig \& Van Melle 1955; Postma 1955). The elastic wave equation corresponding to anisotropic media is costly to solve. To simplify the simulation of anisotropic wave propagation, the weak-anisotropy approximation has been utilized (Thomsen 1986). So to reduce the computational cost, Alkhalifah (2000) derived an acoustic wave equation in transversely isotropic media with a vertical symmetry axis (VTI media) based on the acoustic dispersion relation. He sets the shear wave velocity along the symmetry axis to zero, which results in a fourth-order differential equation (Alkhalifah 1998). Zhou et al. (2006a) introduced an auxiliary parameter to simplify the fourth-order differential equation to a coupled system of second-order partial differential equations. The resulting new acoustic VTI wave equations are easier to solve and adapt to RTM and FWI applications than the original fourth order formula (Duveneck et al. 2008; Duveneck \& Bakker 2011; Song \& Alkhalifah 2020a). Tilted transversely isotropy (TTI) is 
more general to describe the anisotropy of the tilted layered structure of the Earth. Zhou et al. (2006b) further extend their work from VTI to TTI media. They naturally incorporate the phase angle with respect to the symmetry axis in the coupled system of equations and use a cross-derivative term to describe the TTI characteristics. The anisotropy of the Earth has gained more and more attention in seismic imaging and inversion, especially during the last two decades, thanks to the rapid improvements in our computing capabilities.

Numerical methods, such as finite difference (FD) and finite element (FE), have been developed to solve wave equations for different media. However, these methods require significant modifications to the wave propagators when additional physical parameters are considered, which is time-consuming for developers. Furthermore, anisotropic acoustic wave equations for both VTI and TTI media suffer from the shear wave artifacts associated with the Laplacian operator using an FD solver, especially when the sources are located in the anisotropic region (Alkhalifah 2000). These artifacts will introduce huge errors in seismic imaging and inversion. In addition, it is difficult for FD methods to handle models with irregulars shapes, like velocity models with irregular topography. To address these issues, we need to seek an alternative solution to frequency-domain wavefields, especially for anisotropic media.

Neural networks (NNs) have long been recognized as universal approximators using a surfficient number of hidden layers and neurons (Hornik et al. 1989; Leshno et al. 1993; Van der Baan \& Jutten 2000). Thanks to the explosive growth of the available data and the recent developments in computing resources, ANs have become more and more widely used in geophysics, first arrivals and phases of P-and S-waves picking (Dai \& MacBeth 1995; Gentili \& Michelini 2006; Zhu \& Beroza 2019), automatic normal moveout (NMO) correction (Calderón-Mac ı'as et al. 1998), seîsmograms analysis and quality assessment (Valentine \& Trampert 2012), seismic ground-roll noise attenuation (Kaur et al. 2020), automatic seismic source characterization(van den Ende \& Ampuero 2020), direct microseismic event location and characterization (Wang \& Alkhalifah 2021; Wang et al. 2021a), etc. A framework referred to as physics-informed neural networks (PINNs) has been proposed to help us solve partial differential equations (PDEs) (Raissi et al. 2019a). PINNs accept spatial and temporal coordinate values as inputs and uses the underlying physics as the loss function to update the network instead of pure data-mapping objectives. The network, in this case, acts as a universal function approximator, and the partial derivatives with respect to spatial and temporal coordinates can be evaluated using the concept of automatic differentiation. It takes significant efforts for researchers to develop numerical methods to solve different PDEs. By comparison, PINNs have excellent flexibility and versatility for solving any kind of PDEs. We only need to 
modify the loss functions corresponding to the underlying physical laws. In geophysical applications, PINNs have shown its effectiveness in time- and frequency-domain wave equation modelling (Karimpouli \& Tahmasebi 2020; Alkhalifah et al. 2020b), and solving the isotropic and anisotropic P-wave eikonal equations (Smith et al. 2020; Waheed et al. 2020a,b), and magnetotelluric forward modelling (Wang et al. 2021b). Alkhalifah et al. (2020b) used PINNs to solve for the scattered pressure wavefield instead of the whole wavefield directly to avoid the point source singularity, and they also extended their work to VTI media (Song et al. 2021). Though they show the effectiveness and accuracy of solving for the scattered wave equation using PINN, the resulting scattered wavefields are generally smooth, and fail to describe the detailed information of the subsurface. In addition, solving the scattered form of the Helmholtz equation using PINN is only feasible for the wave equations admitting analytical solutions with a point source, which hampers the versatility of this method.

A hyperbolic tangent activation is used to solve a series of PDEs in the original paper introducing PINN and other follow-up research work (Raissi et al. 2019a; Waheed et al. 2020a; Wang et al. 2021c). However, those PDEs tend to have smooth solutions, like the Schrodinger equation, the NavierStokes equation, the eikonal equation equation, etc. Compared to these PDEs, there is another difficulty in solving the Helmholtz equation using PINN. It comes from the sparsity of the source function, and this sparsity will cause a singularity in the wavefield solution, which is hard to represent accurately using neural networks. In this case, the commonly used hyperbolic tangent activation function will perform poorly. Raissi et al. (2019b) argued that the sine activation function is more suitable in learning the vortexinduced vibrations. Sitzmann et al.(2020) recently showed that the sinusoidal representation networks are capable of representing complex signals and their derivatives with high accuracy and resolution. They also shom that the sine-based network can solve time- and frequencydomain wave equations directly. However, their neural networks were large in size requiring costly training. They needed up to 1024 neurons per hidden layer to solve the wave equation for relatively simple models, nothing close to what we encounter in the seismic exploration world. In this paper, we improve their approach by using an adaptive coefficient to initialize the network, and we, thus, refer to our function an adaptive sinusoidal activation function. Using this proposed adaptive initialization scheme, the training convergence can be significantly improved. In addition, we also show that transfer learning will accelerate the training and make the proposed method more practical. We analyse how the network structure affects the wavefield prediction for different models and frequencies. Furthermore, taking advantage of 
the PINNs' features in flexibility and versatility, we further extend the application of solving the Helmholtz equation to anisotropic media.

The paper is organized as follows: After this "introduction", we present the frequencydomain acoustic wave equations for isotropic and anisotropic media and explain how we solve these equations using PINNs. Then, we show numerical results on isotropic and anisotropic models. Finally, we discuss the advantages and limitations of the proposed method.

\section{METHODOLOGY}

\subsection{The isotropic acoustic wave equation}

To simulate acoustic wave propagation, an acoustic wave equation for a constant density medium in the frequency domain is expressed as:

$$
\nabla^{2} p(\mathbf{x}, \omega)+\omega^{2} m p(\mathbf{x}, \omega)=s\left(\mathbf{x}_{\mathbf{s}}, \omega\right)
$$

where $\omega$ is the angular frequency. We use $m=\frac{1}{v^{2}}$ to denote the squared slowness with $v$ denoting the velocity. Here, $p(\mathbf{x}, \omega)$ is the pressure wavefield, and $s(\mathbf{x}, \omega)$ is the source function, in which $\mathbf{x}_{\mathbf{s}}$ represents the spatial location of the source. $\mathbf{x}=\{x, y, z\}$ represents the spatial coordinate in the Cartesian coordinates. $\nabla^{2}:=\frac{\partial^{2}}{\partial x^{2}}+\frac{\partial^{2}}{\partial y^{2}}+\frac{\partial^{2}}{\partial z^{2}}$ is the Laplacian operator. In reality, waves propagate in an infinite domain. However, due tofinite memory of the computer hardware, we often truncate the wavefields into a finite domain of interest using absorbing boundary conditions (Clayton \& Engquist 1977; Higdon 1992) or perfectly matched layer (PML) (Berenger et al. 1994; Hastings et al. 1996; Tsynkov \& Turkel 2001; Chen et al. 2013). Using PML technique to simulate the wavefields within a 2D bounded rectangular domain, the Helmholtz equation is expressed as (Chen et al. 2013):

$$
\frac{\partial}{\partial x}\left(\frac{e_{z}}{e_{x}} \frac{\partial p}{\partial x}\right)+\frac{\partial}{\partial z}\left(\frac{e_{x}}{e_{z}} \frac{\partial p}{\partial z}\right)+e_{x} e_{z} \omega^{2} m p(\mathbf{x}, \omega)=s\left(\mathbf{x}_{\mathbf{s}}, \omega\right)
$$

where

$$
e_{x, z}=1-i \frac{\sigma_{x, z}}{\omega}
$$

$\sigma$ is a differentiable function used to absorb the wave energy within the PML boundaries.

Outside of the PML, $\sigma_{x, z}$ are equal to zero. Meanwhile, inside of the PML, $\sigma_{x, z}$ are defined as:

$$
\sigma_{x, z}=2 \pi a_{0} f_{0}\left(\frac{l_{x, z}}{L_{P M L}}\right)^{2}
$$

where $a_{0}$ is a constant coefficient. Here, we use $a_{0}=0.5 ; f_{0}$ is the dominant frequency of the source function. $L_{P M L}$ represents the physical thickness of PML layers; $l_{x, z}$ represents the 
physical distance from a point $(x, z)$ located inside the PML to the margin of horizontal $(x)$ and vertical $(z)$ PML boundaries. The subscripts $x, z$ in the differentiable function $\sigma$ indicate corresponding to horizontal physical distance $l_{x}$ and vertical physical distance $l_{z}$, respectively. We use the coefficients $A, B, C$ to denote $\frac{e_{z}}{e_{x}}, \frac{e_{x}}{e_{z}}$, and $e_{z} e_{x}$, respectively. Then, eq.2 can be written as:

$$
\frac{\partial}{\partial x}\left(A \frac{\partial p}{\partial x}\right)+\frac{\partial}{\partial z}\left(B \frac{\partial p}{\partial z}\right)+C \omega^{2} m p=s\left(\mathbf{x}_{\mathbf{s}}, \omega\right)
$$

\subsection{The anisotropic acoustic wave equation}

Considering the anisotropic nature of the Earth, the anisotropic acoustic wave equation is often used to simulate wave propagation that approximately represents (at least kinematically) the behavior of P-waves inside the Earth. In acoustic VTI media, we can solve a coupled system of second-order differential equations to get the frequency-domain wavefields. The 2D frequencydomain acoustic VTI wave equation with constant density parameterized using the normal moveout (NMO) velocity $v_{n}$ and the anisotropic parameters $\delta$ and $\eta$, is stated as follows (Zhou et al. 2006a):

$$
\begin{aligned}
& \omega^{2} m_{n} p+\frac{\partial^{2}(p+q)}{\partial x^{2}}+\frac{1}{(1+2 \delta)} \frac{\partial^{2} p}{\partial z^{2}}=s, \\
& \omega^{2} m_{n} q+2 \eta \frac{\partial^{2}(p+q)}{\partial x^{2}}=0
\end{aligned}
$$

where $q$ is an auxiliary perturbation wavefield. We use $m_{n}=\frac{1}{v_{n}^{2}}$ to represent the NMO squared slowness. VTI assumption is valid for simple geologic structures, like shales in flat sedimentary basins. However, it cannot provide a good representation of anisotropy for dipping geologic formations, such as synclines and anticlines (Zhou et al. 2006a; Operto et al. 2009; Waheed \& Alkhalifah 2015). Similar to the acoustic VTI wave equation (Zhou et al. 2006a), a coupled system of second-order differential equations are used in TTI media. In addition, each equation in the coupled system has a cross-derivative term due to its TTI characteristics (Zhou et al. 2006b). The acoustic TTI wave equations are expressed as:

$$
\begin{aligned}
& \omega^{2} m_{0} p+(1+2 \delta) H p+H_{0} p+(1+2 \delta) H q=s, \\
& \omega^{2} m_{0} q+2(\epsilon-\delta) H q+2(\epsilon-\delta) H p=0, \\
& H=\cos ^{2} \theta_{0} \frac{\partial^{2}}{\partial x^{2}}+\sin ^{2} \theta_{0} \frac{\partial^{2}}{\partial z^{2}}-\sin 2 \theta_{0} \frac{\partial^{2}}{\partial x \partial z}, \\
& H_{0}=\sin ^{2} \theta_{0} \frac{\partial^{2}}{\partial x^{2}}+\cos ^{2} \theta_{0} \frac{\partial^{2}}{\partial z^{2}}+\sin 2 \theta_{0} \frac{\partial^{2}}{\partial x \partial z},
\end{aligned}
$$


where $\theta_{0}$ is the angle of the symmetry axis with respect to the $z$ axis. $m_{0}$ represents the squared slowness along the symmetry axis. In acoustic anisotropic wave equation for TTI media, Thomsen dimensionless parameters $\epsilon$ and $\delta$ are used to describe anisotropy (Thomsen 1986).

\subsection{The physics-informed neural networks}

We consider a fully connected DNN consisting of $L+1$ layers, starting with input layer 0 , the output layer $L$, and $L-1$ hidden layers, to approximate a function $u(x, z)$. In the $l$-th hidden layer, the number of neurons is denoted as $k^{l}$. The $i$-th neuron in layer $l-1$ and $j$-th neuron in layer $l$ is connected by a weighting parameter defined as $w_{j i}^{l}$. Each neuron contributes to the network by taking a weighted sum of inputs in layer $l-1$, and a bias term, defined by $b$. In a feed-forward network, the output for the $k$-th neuron in next layer $l$ is given by (Bishop 2006):

$$
u_{k}^{l}=\sigma\left(\sum_{j=1}^{k_{l}-1} w_{k j}^{l} u_{j}^{l-1}+b_{k}^{l}\right),
$$

where $\sigma()$ denotes the activation function. We feed spatial coordinate values to the network as the input data. The output parameters of the network are the real and imaginary parts of the complex pressure wavefield $p$ for isotropic media. While, for anisotropic media, we also include the real and imaginary parts of auxiliary wavefield as the output parameters. Taking advantage of the concept of automatic differentiation (Baydin et al. 2017), the second-order derivatives with respect to spatial coordinates can be easily evaluated. Thus, to train the network to provide wavefields that satisfy the isotropic acoustic wave equation, we use the following loss function:

$$
f=\frac{1}{N} \sum_{i=1}^{N}\left|\frac{\partial}{\partial x}\left(A^{(i)} \frac{\partial p^{(i)}}{\partial x}\right)+\frac{\partial}{\partial z}\left(B^{(i)} \frac{\partial p^{(i)}}{\partial z}\right)+C^{(i)} \omega^{2} m^{(i)} p^{(i)}-s^{(i)}\right|_{2}^{2},
$$

in which we minimize the physics-constrained mean squared error (MSE). $i$ and $N$ represent the training pøínts index and training points number within the domain of interests. The squared slowness $m$, frequency information $\omega$, PML coefficients $A, B$, and $C$ are non-trainable parameters used in the loss function, and the values of which are corresponding with the input coordinate values. These parameters are only incorporated in the loss function, so they are not used as input for the test data. Similar to solving the isotropic wave equation, we can use the anisotropic wave equation in eqs. 6 and 7 as the loss function to train the network and lead to wavefield solutions considering the anisotropy. In all the experiments shown in this paper, an Adam optimizer and a follow-up L-BFGS optimization, which is a quasi-Newton 
approach, with full-batch are used to optimize the loss function (Liu \& Nocedal 1989). From our experiments, an Adam optimizer followed by an L-BFGS optimization is the best strategy for training. Through trials, starting with the L-BFGS optimizer alone will causes slow convergence. However, if we use the Adam optimizer first, and then, the follow-up L-BFGS will accelerate the convergence. Using the framework of PINN, it is very easy to adapt for more complex media, like the acoustic anisotropic media mentioned above. We can easily transform eqs. 6 and 7 to the form of loss functions used in PINN like the form of eq. 10. By comparison, it needs tremendous modifications to convert the conventional numerical solvers from isotropic media to anisotropic media.

\subsection{Adaptive sinusoidal activation function}

The activation function $\sigma()$ used in DNN has a large influence on training and predictions of the network. There are a variety of commonly used activation functions, such as the logistic sigmoid, the rectified linear unit $(\operatorname{ReLU}(x))$, the hyperbolic tangent $(\tanh (x))$, and the inverse tangent $(\operatorname{atan}(x))$ (Sibi et al. 2013). ReLU $(x)$ and many variation forms of ReLU activation functions, like ParametricReLU $(x)$, LeakyReLU $(x)$, are popularly used and well studied. However, they are piecewise linear, and their second derivative is a constant value, which equals zero. As a result, they cannot be used to represent a function containing spatial and temporal derivatives higher than second order. In this paper, we use $\sigma(x)=\sin (x)$ (Raissi et al. 2019b; Sitzmann et al. 2020). As we mentioned above, the input data to PINN are the spatial coordinate values. Using the sine activation function, eq.9 for layer 1 can be written as:

$$
u^{1}=\sin \left(W^{1} \mathbf{x}+b^{1}\right) .
$$

We use $W^{1}$ to represent the matrix of weights connecting layer 0 and 1 and $b^{1}$ to represent the vector of bias in layer 1. $\mathrm{x}$ represents the input spatial coordinate in layer 0. Mathematically, $W^{1}$ acts as the spatial wavenumber $k_{x}$ and $k_{z}$ for $x$ and $z$ directions for a plane wave, and $b^{1}$ acts as the phase shift for a sine function signal. Acoustic waves can be represented by weighted sine signals with different wavenumbers (i.e. inverse spatial Fourier transform). The range of $k_{x}$ and $k_{z}$ is between $-\frac{\omega}{v_{\text {min }}}$ and $\frac{\omega}{v_{\text {min }}}$ between layer 0 and layer 1 to properly represent our waves. For the other layers the sine function with small weights act like linear functions to combine the plane waves generated in the first layer to represent the resulting wavefield. The weights in all the hidden layers are initialized in the same way using a Xavier Initialization with a uniform distribution (Glorot \& Bengio 2010). In the work of Sitzmann et al. (2020), 
they use a fixed coefficient $w_{0}$ to increase the weights between layer 0 and layer 1 in the sine network, which is stated as $u^{1}=\sin \left(w_{0} \cdot W^{1} \mathbf{x}+b^{1}\right)$. We refer to this setup as a fixed $\sin (x)$ activation function. In this paper, we propose to optimize this coefficient $w_{0}$ in the training process to help achieve better performance of the network (Shukla et al. 2020), and we define this technique as the adaptive $\sin (x)$ activation function. This can be easily implemented in TensorFlow. The adaptive coefficient in $\sin (x)$ can be defined as a Variable, and it can be initialized and updated in the training process.

\subsection{Network training}

To test the performance of PINN, we use fully-connected DNNs with 8 hidden layers, and each hidden layer has 40 neurons. The PINN architectures of wavefields predictions for the isotropic and anisotropic media are shown in Fig. 1a and 1b, respectively. The inputs are given by the spatial coordinates of points in the model in both cases. For the isotropic case, the outputs are given by the real and imaginary parts of the pressure wavefield at these points; while for the anisotropic case, the outputs are given by the real and imaginary parts of the pressure and auxiliary perturbation wavefields. We borrow the PINN architecture from previous work (Raissi et al. 2019a; Alkhalifah et al. 2020b; Song et al. 2021), whích proves to be efficient and effective. In all the experiments shown in this paper, an Adam optimizer and a follow-up L-BFGS optimization, which is a quasi-Newton approach, with full-batch are used to optimize the loss function (Liu \& Nocedal 1989).

To illustrate the advantages of the proposed adaptive sine activation function, we compare it with $\tanh (x)$ and the fixed $\sin (x)$ activation functions using the same training setup. The computational cost for these three activation functions is almost the same.

The hardware we use to train the networks is a Quadro RTX 8000 GPU with 48 GB of memory, and we implement the proposed method using TensorFlow (Abadi et al. 2015).

\section{RESULTS}

In this section, we share the results from implementing the proposed method on a set of isotropic and anisotropic models. We compare PINN-predicted results with numerical wavefield solutions from an optimal 9-point FD wave equation operator (Jo et al. 1996). In all the examples, the source function is given by an $8 \mathrm{~Hz}$ Ricker wavelet, which is a representative frequency for FWI. We use an isotropic Gaussian source with a variance of 3 instead of a point source to reduce the source singularity to some degree. In the frequency-domain wave- 
(a)

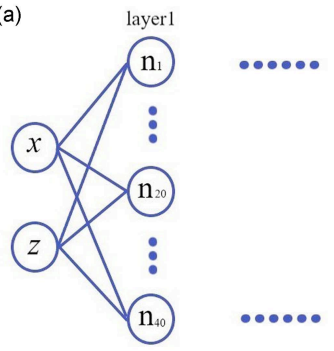

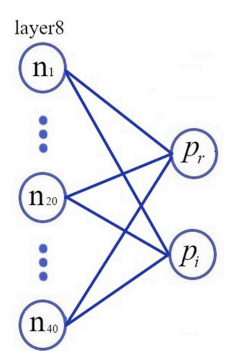
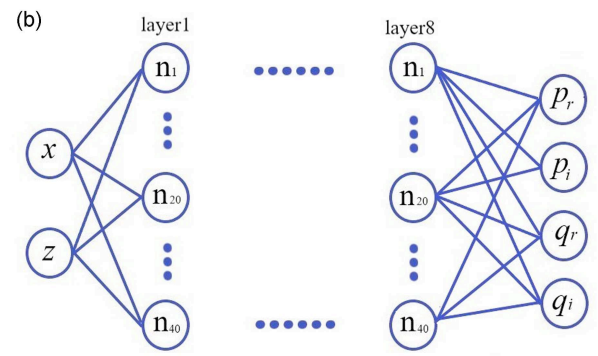

Figure 1. The PINN architecture for wavefields predictions in (a) the isotropic media and (b) anisotropic media. For both cases, the inputs are given by the spatial coordinates of points in the model. For the isotropic case, the outputs are given by the real and imaginary parts of the pressure wavefield at these points; while for the anisotropic case, the outputs are given by the real and imaginary parts of the pressure and auxiliary perturbation wavefields.

field modeling, the time-domain Ricker wavelet is transformed into a group of complex values. For a specific frequency, we multiply the Gaussian source with the corresponding complex valued Fourier representation of the Ricker wavelet.

\subsection{An isotropic homogeneous model}

First, we use an isotropic homogeneous model to test the proposed method. The velocity is set to $2 \mathrm{~km} / \mathrm{s}$. The size of the model is $101 \times 101$ with a grid interval of $20 \mathrm{~m}$ in both the vertical and horizontal directions. For all the models used in this paper, we extend the physical model by 50 grid points along all the boundaries to form the PML, which is considerably large, and necessary to guarantee the elimination of boundary reflections. We first show the real and imaginary parts of the numerical wavefield solution corresponding to a source in the center of the model for $4 \mathrm{~Hz}$ in Firs. 2a and 2b, respectively. The areas inside of the black frames indicate the physical domains of interest, while the areas outside of the black frames indicate the PML. We observe that the wave energy is well absorbed by the PML boundaries.

We randomly select three quarters of all the regular grid points (including the points in the PML boundaries) and feed them to the network. We use 50,000 epochs of Adam optimizer and 50,000 E-BFGS updates. The runtime for each epoch of Adam training and each iteration of L-BFGS optimization is $0.072 \mathrm{~s}$ and $0.062 \mathrm{~s}$, respectively. We show the loss function curves for the Adam training of the NN for different activation functions in Fig. 3a. It is obvious that NN with $\tanh (x)$ activation function fails to achieve convergence in the training. By comparison, the $\sin (x)$ activation function can converge generally well in the Adam optimizer training, 

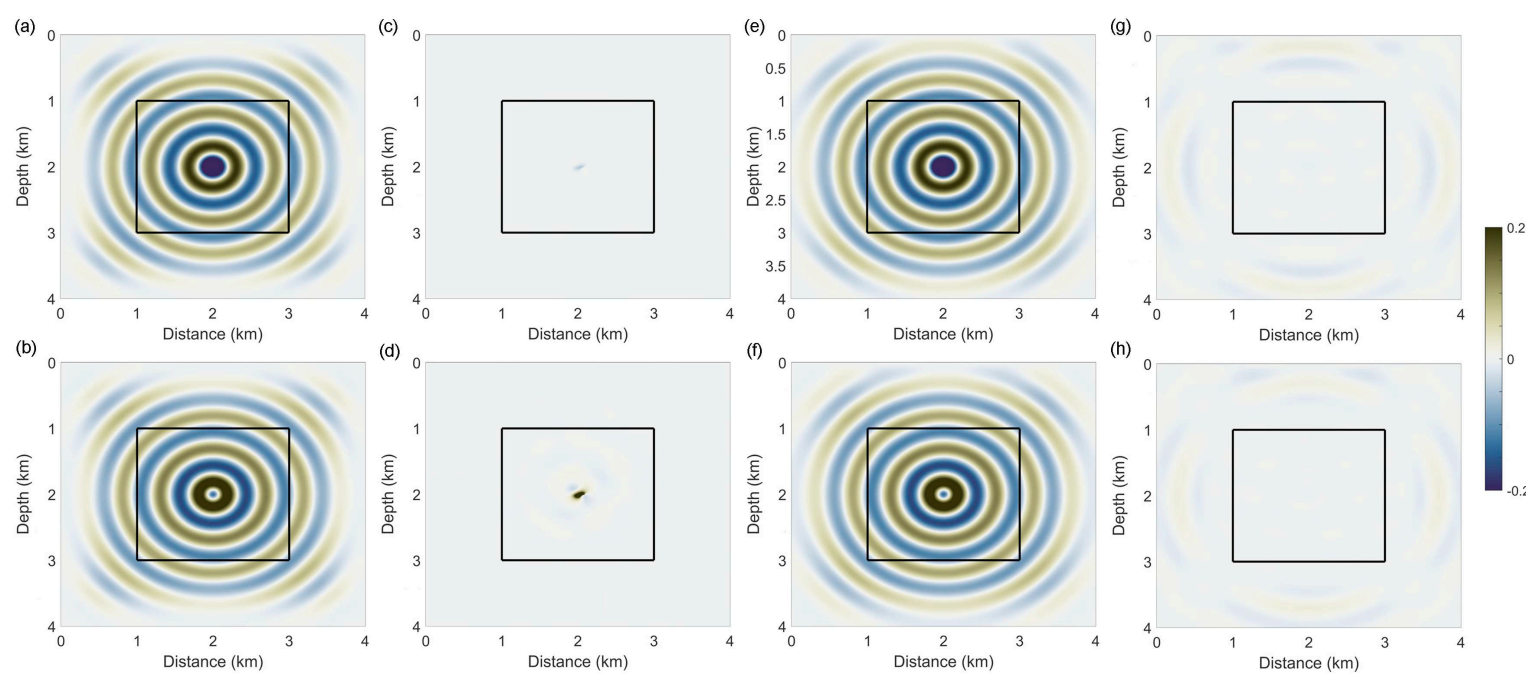

Figure 2. The real and imaginary parts of the $4 \mathrm{~Hz}$ wavefields (a,b) from the finite-difference method, $(\mathrm{c}, \mathrm{d})$ from PINN with the $\tanh (x)$ activation function, $(\mathrm{e}, \mathrm{f})$ from PINN with the adaptive $\sin (x)$ activation function for the homogeneous model, and $(g, h)$ wavefield difference between $(a, b)$ and $(e, f)$.

and NN with adaptive $\sin (x)$ accelerates the converging rate. Fig. 3b shows the variation of the updated $w_{0}$ Adam training epochs.

We observe a rapid increase of $w_{0}$ in the early stage of training, then $w_{0}$ tends to stabilize for this model around 5.5. We first show the real and imaginaryparts of the predicted wavefield solution using PINN with $\tanh (x)$ activation function in Figs. 2c and 2d, respectively. As we fail to train the network well using $\tanh (x)$, the output wavefield is clearly a wrong solution.

(a)

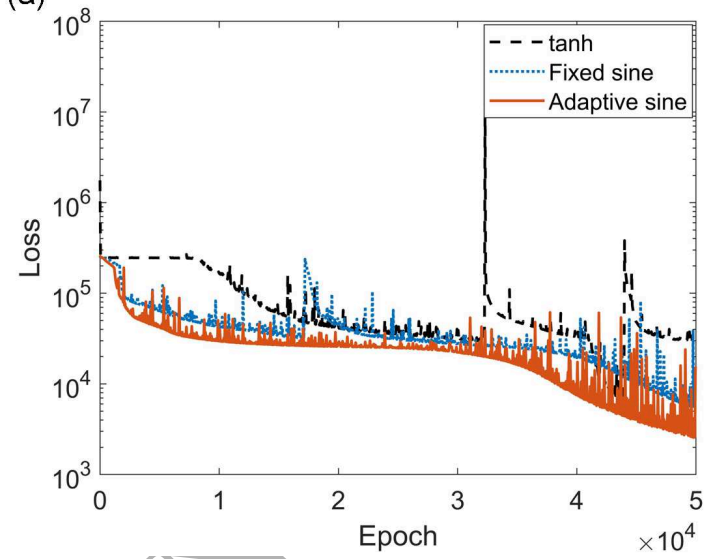

(b)

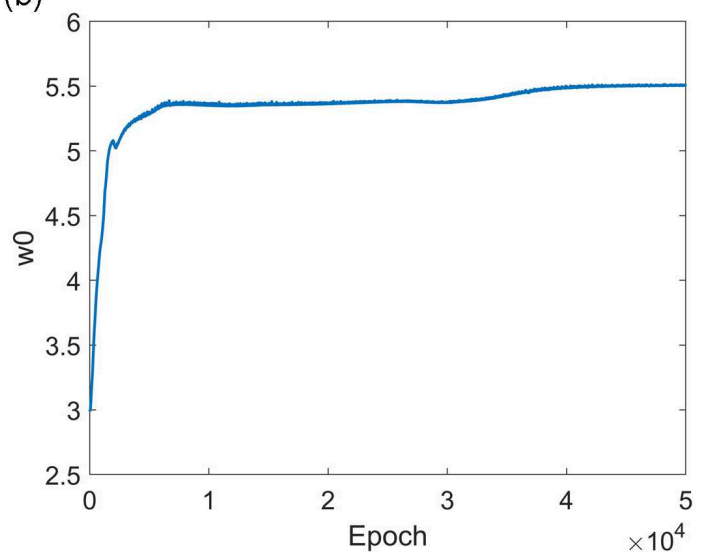

Figure 3. (a) The loss function for the training of the NN for different activation functions, and (b) the $w_{0}$ variation for the Adam training. In (a), the black dashed curve corresponds to the $\tanh (x)$ activation function; the blue dotted curve corresponds to the fixed $\sin (x)$ activation function; the red solid curve corresponds to the adaptive $\sin (x)$ activation function. 
Next, we show the real and imaginary parts of the predicted wavefield solution using PINN with an adaptive sine activation function in Figs. 2e and 2f, and the results are considerably improved. To compare this wavefield solution with the numerical one, we show their differences in Figs. $2 \mathrm{~g}$ and $2 \mathrm{~h}$. The differences are mainly distributed in the PML boundaries, while we can hardly see any difference in the physical domain (inside the black frame). We save this trained network as a starting network for another velocity model (seen in the next subsection) to accelerate the training convergence using the concept of transfer learning (Pan \& Yang 2009; Waheed et al. 2021).

We next discuss how should we adjust the training setup to solve for higher-frequency wavefield solutions. For $8 \mathrm{~Hz}$, we show the real part of the wavefield solution from the numerical method in Fig. 4a. The sinusoidal nature of the wavefield becomes more obvious as the frequency increases. If we still use three quarters of regular grid points as input data and 50,000 epochs of Adam optimizer and 50,000 L-BFGS updates with adaptive $\sin (x)$ activation function, we obtain a wavefield with the real part of it shown in Fig. 4b. We fail to get a reasonable PINN-predicted wavefield even with the adaptive $\sin (x)$. To improve the PINN result, we use all the regular points as input data and the same training setup as above to train the network. All the grid points are arranged in random order before we feed them to the network. The real part of the resulting wavefield solution is shown in Fig. 4c. We observe that the general shape of the wavefield is recovered, but with a weaker amplitude. To further improve the network, we increase the Adam trainîng epochs to 150,000. After such sufficient training, the real part of the predicted wavefield is Fig. 4d. The output result is reasonably good and resembles the numerical solution in Fig.4a. We show the difference in Fig. 4e, which is quite small. By comparison, the wavefield solution from PINN with $\tanh (x)$ using all the regular points and 150,000 epochs of Adam optimizer is show in Fig. 4f. Although PINN with $\tanh (x)$ can provide a reasonable result, it is inferior to the result obtained from PINN with the adaptive $\sin (x)$.

\subsection{The isotropic Marmousi model}

Next, we consider a more complicated and realistic model to test the proposed method. The isotropic Marmousi model we use in this example is shown in Fig. 5a, and the model size is $371 \times 101$. The horizontal and vertical space sampling interval is $25 \mathrm{~m}$. We place the source on the surface at location $4.625 \mathrm{~km}$. We first show the real part of the numerical wavefield solution for $3 \mathrm{~Hz}$ in Fig. 5b. In this example, and all following examples, we just show the wavefield solutions in the physical domain and ignore the areas within PML. 

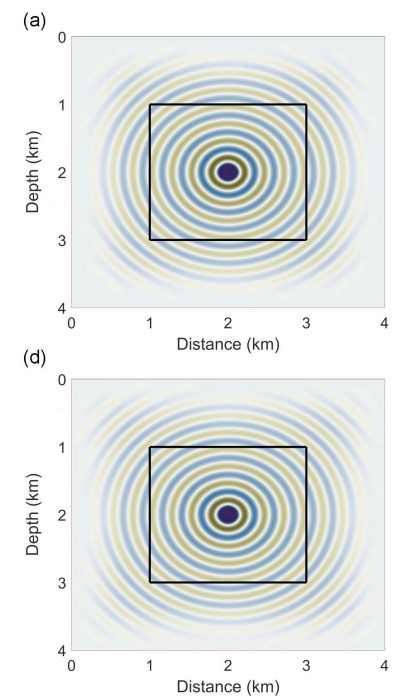
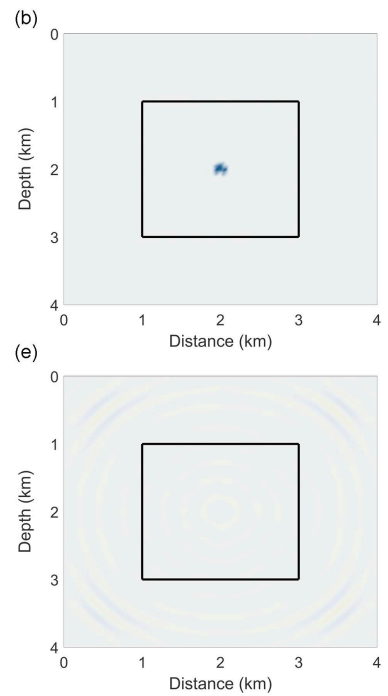

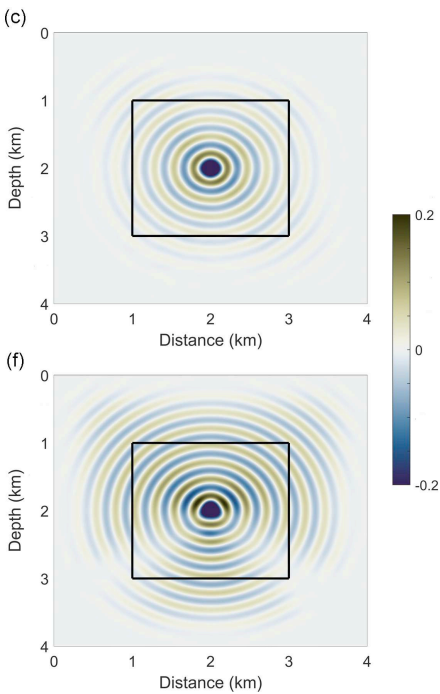

Figure 4. The real part of the wavefield solution from (a) the conventional numerical method, (b) PINN with adaptive $\sin (x)$ using three quarters of the regular points and 50,000 epochs of Adam optimizer, (c) PINN with adaptive $\sin (x)$ using all the regular points and 50,000 epochs of Adam optimizer, (d) PINN with adaptive $\sin (x)$ using all the regular points and 150,000 epochs of Adam optimizer, (e) difference between Figs. 4a and 4d, and (f) PINN with $\tanh (x)$ using all the regular points and 150,000 epochs of Adam optimizer.

We still use the network in Fig. 1a for this example. We yandomly input all the regular grid points to the network and use 100,000 epochs of Adam optimizer and 50,000 L-BFGS updates to train the network using different activations. As this model is far more complicated than the previous homogeneous one, it requires a larger number of Adam training epochs. The runtime for each epoch of Adam training and each iteration of L-BFGS optimization is $0.18 \mathrm{~s}$ and $0.15 \mathrm{~s}$, respectively. It is 2.5 times the case for the homogeneous model. This

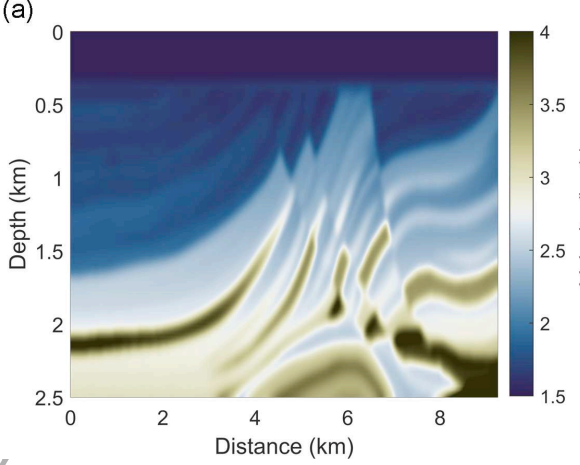

(b)

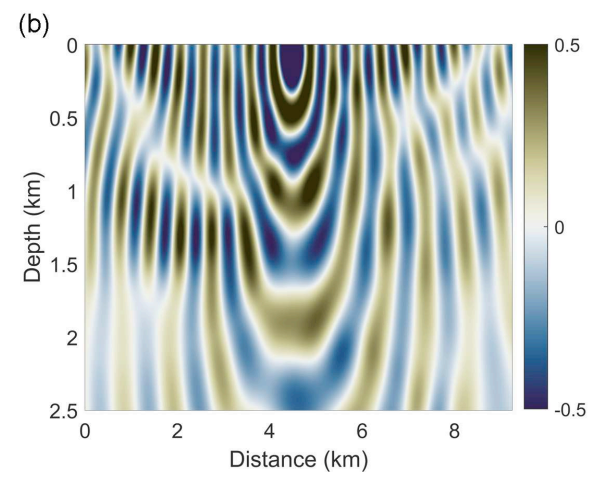

Figure 5. (a) The isotropic Marmousi model, and (b) the real part of the numerical wavefield solution corresponding to it at $3 \mathrm{~Hz}$ using an FD solver. 


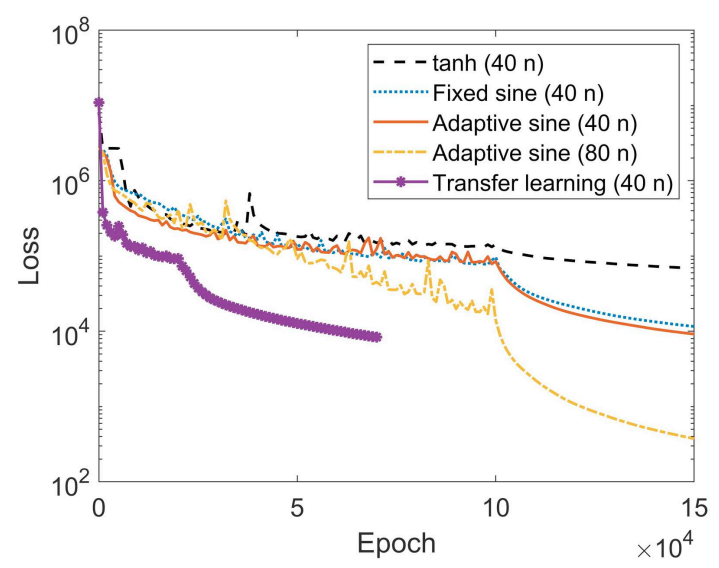

Figure 6. The loss function for the training of the NN corresponding to different activation functions The black dashed curve corresponds to the $\tanh (x)$ activation function; the blue dotted curve corresponds to the fixed $\sin (x)$ activation function; the red solid curve corresponds to the adaptive $\sin (x)$ activation function; the yellow dashed dotted curve corresponds to the adaptive $\sin (x)$ activation function with 80 neurons in each hidden layer; the purple star solid curve corresponds to the adaptive $\sin (x)$ activation function using the transfer learning.

number is a lot smaller than the theoretical cost we will have for a numerical solver as the Marmousi model size is about three times as large as the homogeneous model. Theoretically, the computational cost for the Marmousi model should be $3^{3}=27$ times that for the case the homogeneous model using the finite-difference method. In this example, we show the loss function curves for both Adam and L-BFGS training corresponding to different activation functions in Fig. 6. Compared to the $\tanh (x)$ activation function, PINN with a fixed $\sin (x)$ activation function can converge to a lower training loss, while the adaptive $\sin (x)$ can help PINN reach an even lower loss. If transfer learning is applied by starting with the previously trained network, we observe a rapid training decrease in loss at the beginning of the training. In this case, only 20,000 Adam epochs are sufficient to achieve convergence. The sudden loss decrease in each curve at 100,000-th epoch comes from the optimizer transmission from the ADAM to the L-BFGS. For the transfer learning case, the sudden loss decrease occurs at 20,000-th epoch, ass shown in Fig. 6.

We show the PINN-predicted wavefields based on the trained model at the last epoch. Using $\tanh (x)$ activation function, the real part of the PINN-predicted wavefield solution is shown in Fig. 7a. It is obvious that the wavefield solution using PINN with $\tanh (x)$ can only recover the wavefield below the source location, and we observe a large wavefield difference between the numerical wavefield solution and the PINN-predicted wavefield solution in Fig. 7b. 

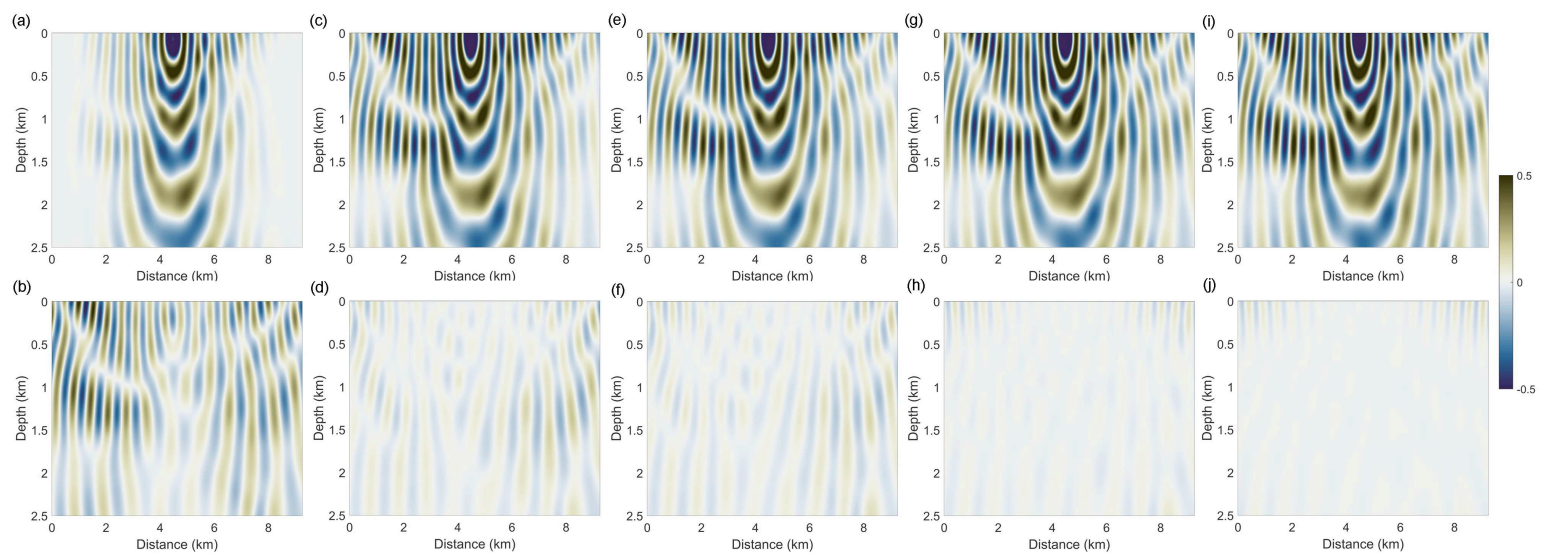

Figure 7. The $3-\mathrm{Hz}$ real part of the PINN-predicted wavefield solution (a) from the $\tanh (x)$ activation function, (c) from the fixed $\sin (x)$ activation function, (e) from the proposed adaptive $\sin (x)$ activation function, $(\mathrm{g})$ from the adaptive $\sin (x)$ activation function considering the transfer learning, (i) from the adaptive $\sin (x)$ activation function using the network with 80 neurons in each hidden layer for the Marmousi model. The wavefield difference (b) between $5 \mathrm{~b}$ and $7 \mathrm{a},(\mathrm{d})$ between $5 \mathrm{~b}$ and $7 \mathrm{c},(\mathrm{f})$ between $5 \mathrm{~b}$ and $7 \mathrm{e},(\mathrm{h})$ between $5 \mathrm{~b}$ and $7 \mathrm{~g},(\mathrm{j})$ between $5 \mathrm{~b}$ and $7 \mathrm{i}$.

We calculate the $l_{2}$ norm of the wavefield difference, which is 32.3. Or the other hand, the PINN-predicted wavefield solution using a fixed $\sin (x)$ activation function in Fig. $7 \mathrm{c}$ is able to retrieve the wavefield solution in the whole domain. We show the wavefield difference between the numerical wavefield solution and PINN-predicted wavefield solution using the fixed $\sin (x)$ in Fig. $7 \mathrm{~d}$, and it is much smaller than the wavefield difference in Fig. 7b. The $l_{2}$ norm of the wavefield difference is 16.0, which is half of the case for using $\tanh (x)$. Then, we try to use the proposed adaptive $\sin (x)$ to train the network, and the resulting wavefield solution is shown in Fig. 7e. It is difficult to visually distinguish the wavefield solutions from fixed and adaptive $\sin (x)$ activation functions. However, the $l_{2}$ norm of the wavefield difference (Fig. 7f) is 15.0, and it is quantitatively smaller than the case for a fixed $\sin (x)$, which confirms the superiority of the adaptive $\sin (x)$. Finally, we show the resulting wavefield solution using the transfer learning technique ín Fig. 7g. It looks very close to the numerical solution. The wavefield difference is quite small, as shown in Fig. 7h. For this case, the $l_{2}$ norm of the wavefield difference 13.9. This wavefield residual is smaller than the previous cases, and it confirms the efficiency and accuracy of the adaptive $\sin (x)$ using transfer learning.

To show the effect of the network size on the results, we increase the neurons in each hidden layer from 40 to 80. Training the network using 100,000 epochs of Adam optimizer and 50,000 L-BFGS updates with all the regular grid points, we observe that the training loss will decrease to a much lower loss than the cases for 40 neurons in each hidden layer, as 

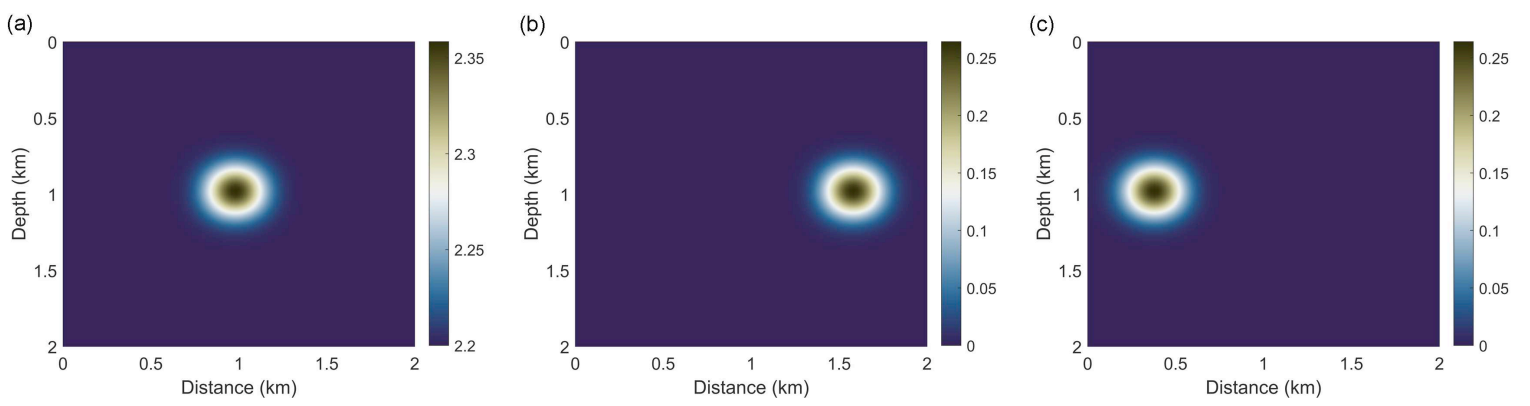

Figure 8. The (a) true velocity, (b) $\delta$, and (c) $\eta$ for the VTI anomaly model.

shown in Fig. 6. The resulting PINN-predicted wavefield solution using the adaptive $\sin (x)$ activation function is shown in Fig. 7i. We observe very small wavefield difference between the numerical solution (Fig. 7j) and PINN-predicted wavefield solution (Fig. 7a). Quantitatively, the $l_{2}$ norm of the wavefield difference is 12.1 , which is the smallest among all the experiments for the example. It shows that this larger network is more expressive to capture the complexity of the resulting wavefield. However, the computational cost for this larger network is almost as twice as high as the smaller one.

\subsection{A VTI anomaly model}

We further apply the proposed method on a VTr anomalies model. The true velocity, $\delta$, and $\eta$ anomalies are located at different locations laterally in a homogeneous isotropic background model, as shown in Figs. 8a-8c, respectively. The size of the models is $101 \times 101$ with a grid interval of $20 \mathrm{~m}$ in both the vertical and horizontal directions. We place the source on the surface of the model located at $1 \mathrm{~km}$. In VTI media, the pressure wavefield is our main concern, so we only show the real and imaginary parts of the $6 \mathrm{~Hz}$ pressure wavefield using a numerical method in Figs. 9a and 9b, respectively. In the circled area, we can clearly see that the anisotropic parámeter $\delta$ anomaly has effected the wave propagation.

For this VTI anomalies model, we train the network in Fig. 1b using 100,000 epochs of Adam optimizer and 50,000 L-BFGS updates. For this anisotropic model, the runtime for each epoch of Adam training and each iteration of L-BFGS optimization is $0.11 \mathrm{~s}$ and 0.09 $\mathrm{s}$, respeetively. For the same model size, the computational cost of the numerical solver for anisotropic media is 8 times that for isotropic media (Song et al. 2021). While PINN only adds a limited extra cost when we extend from isotropic media to anisotropic media. The real and imaginary parts of the PINN with $\tanh (x)$ predicted wavefield are shown in Figs. 9c 

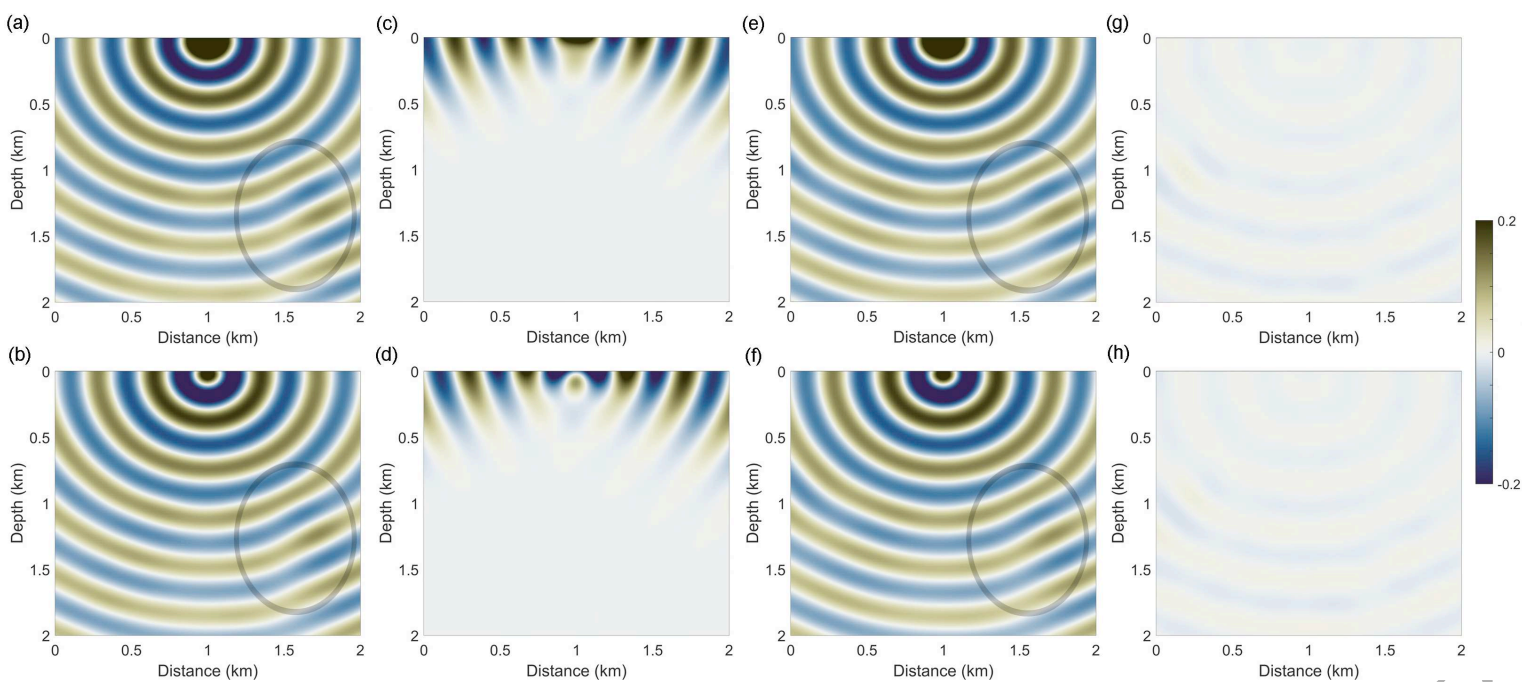

Figure 9. The real and imaginary parts of the $6 \mathrm{~Hz}$ wavefields (a,b) from the finite-difference methød, $(\mathrm{c}, \mathrm{d})$ from PINN with $\tanh (x)$ activation function, $(\mathrm{e}, \mathrm{f})$ from PINN with $\sin (x)$ activation function for the VTI anomalies model, and $(\mathrm{g}, \mathrm{h})$ wavefield difference between figure $(\mathrm{a}, \mathrm{b})$ and $(\mathrm{e}, \mathrm{f})$. (Circled areas indicate the anisotropic signature.)

and 9d, respectively. We observe that PINN with $\tanh (x)$ fails to generate reasonable wavefield solutions.

Using the adaptive $\sin (x)$ activation function, the real and imaginary parts of the PINNpredicted pressure wavefield are shown in Figs. 9e and 9f, respectively. The results demonstrate that PINN with adaptive $\sin (x)$ can retrieve a reasonable wavefield solution with the anisotropic signature, as shown in the circled areas in Fig. 9e and 9f. We show the real and imaginary parts of the wavefield difference between the numerical and PINN with the adaptive $\sin (x)$ methods in Figs. $9 \mathrm{~g}$ and $9 \mathrm{~h}$, respectively. We observe that the wavefield difference is very small, which indicates the effectiveness of PINN with the adaptive $\sin (x)$ in solving the acoustic wave equation for XTI media.

\subsection{A TTI homogeneous model}

To further show the validity the proposed method in solving the acoustic wave equation for TTI media, we consider anisotropic parameters with $v_{0}=2.2 \mathrm{~km} / \mathrm{s}, \epsilon=0.2$, and $\delta=0.05$, with different $\theta_{0}$. The size of the model is $101 \times 101$, and the grid interval for both vertical and horizontal directions is $20 \mathrm{~m}$. For a $6 \mathrm{~Hz}$ wavefield with the source in the center of the model, we use the network in Fig. 1b with adaptive $\sin (x)$, and we train it using 50,000 epochs of Adam optimizer and 50,000 L-BFGS updates. The real part of the PINN-predicted wavefields corresponding to $0^{\circ}, 45^{\circ}, 90^{\circ}$, and $-45^{\circ}$ angles of the symmetry axis are shown in Figs. 10a- 
(a)

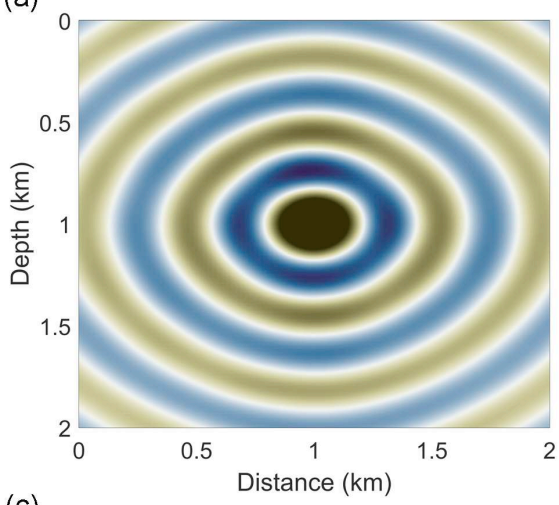

(c)

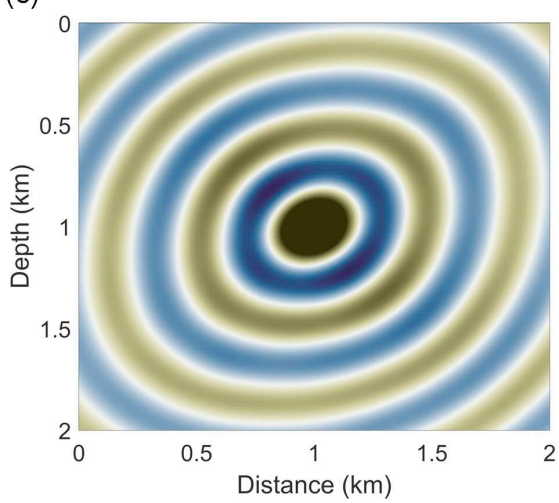

(b)

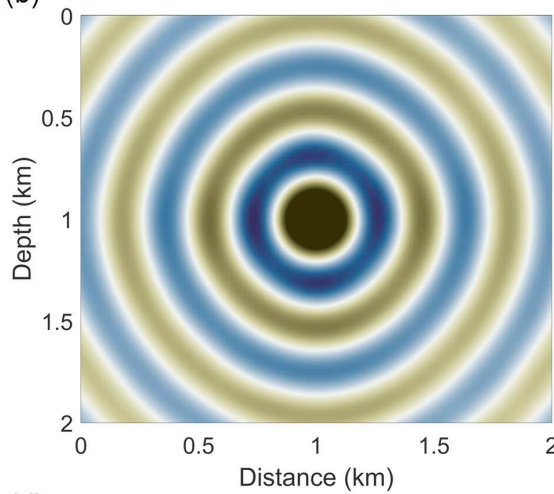

(d)

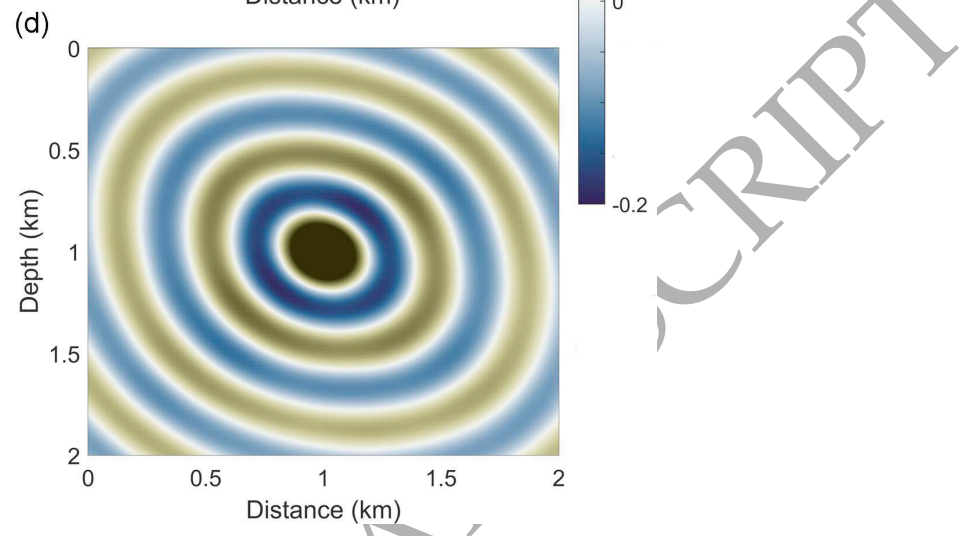

Figure 10. The real part of the 6-Hz PINN-predicted wavefield in TTI media with the angle of the symmetry axis being (a) $0^{\circ}$, (b) $45^{\circ}$, (c) $90^{\circ}$, and (d) $-45^{\circ}$.

10d, respectively. The wavefields show that waves propagate slower along the symmetry axis, as expected with the considered model parameters. Even though the source is located in the anisotropic region, the wavefield solution does not suffer from shear wave artifacts, which often occur in the FD modelling (Alkhalifah 2000; Zhou et al. 2006a,b).

\subsection{A TTI model with topography}

Finally, we apply this method on a model with irregular topography, as shown in Fig. 11. The size of the model is $201 \times 101$ with $20 \mathrm{~m}$ spatial sampling interval in both the vertical and horizontal directions. We place the source in the center at $(2.5 \mathrm{~km}, 1.25 \mathrm{~km})$, and we ignore the influence of the free-surface condition. We first ignore the anisotropy of the model and solve for a $5 \mathrm{~Hz}$ isotropic wavefield. We input the grid points located below the topography to the network. Using the same network as the one used in the previous example, we use the adaptive $\sin (x)$ activation function to train this network. After 200,000 epochs of Adam optimizer and 50,000 L-BFGS updates, we show the real and imaginary parts of the PINNpredicted wavefield in Figs. 12a and 12b, respectively. 


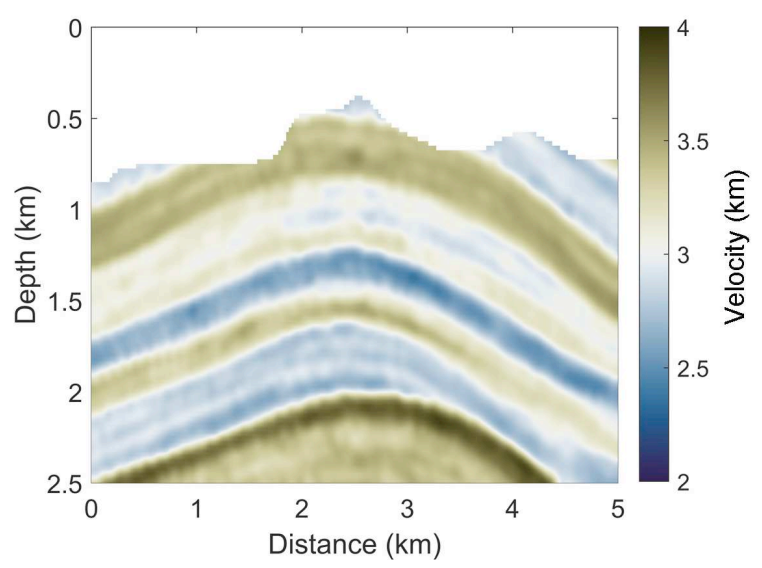

Figure 11. The true velocity with irregular topography.

To verify the accuracy of the PINN-predicted pressure wavefield, we calculata the velocity using the PINN-predicted wavefield according to eq. 1, and show it in Fig. 13. We observe that the predicted velocity reconstructs all the details in the true topography model, except for the source location usually affected by the source singularity.

Then, we build $\epsilon$ and $\delta$ models by dividing the true velocity model by a constant value, as shown in Figs. 14a and 14b, respectively. With the same network architecture and training setup used in the isotropic topography model, we predict the wavefield solution corresponding to the anisotropic topography models and a $45^{\circ}$ angle of the symmetry axis, and the real and imaginary parts are shown in Figs. $15 \mathrm{a}$ and 156 , respectively. Compared to the wavefield in Fig. 12 corresponding to the isotropic topography model, we clearly can see that the anisotropic wavefield in Fig. 15 is skewed towards the symmetry axis.

(a)

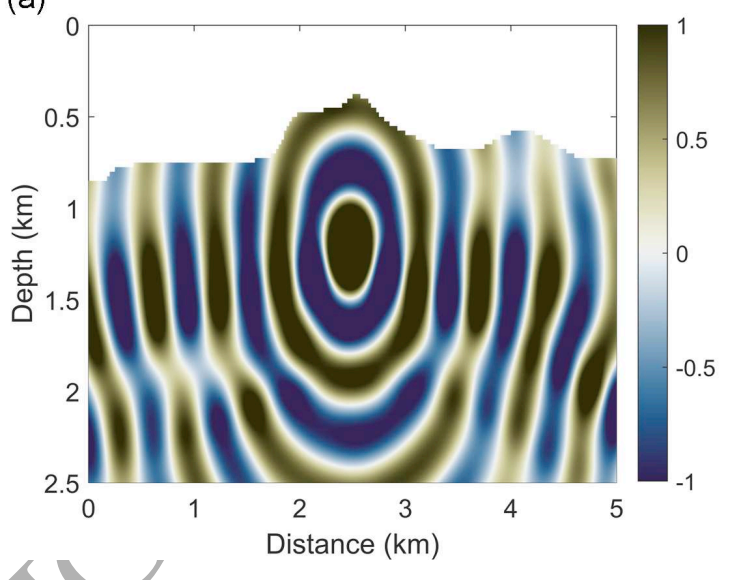

(b)

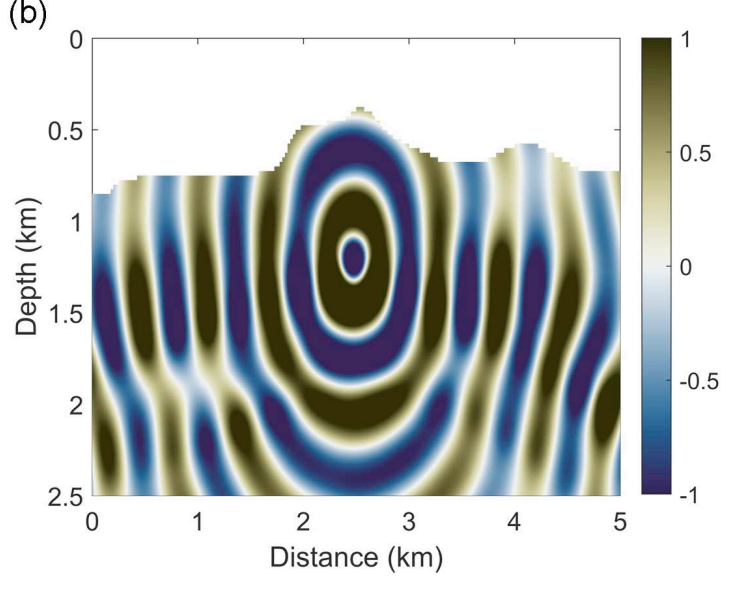

Figure 12. The (a) real and (b) imaginary parts of the PINN-predicted pressure wavefield solution with adaptive $\sin (x)$ activation function for the isotropic topography model of $5 \mathrm{~Hz}$. 


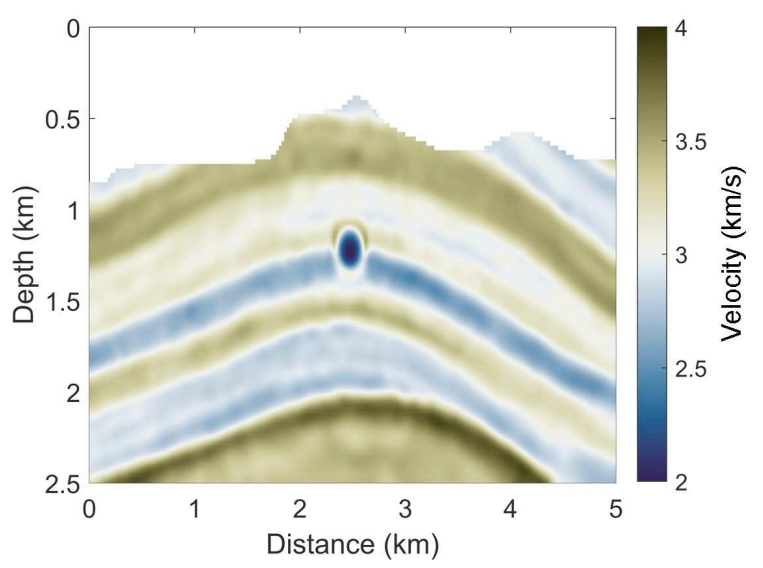

Figure 13. The predicted topography model using PINN-predicted wavefield in Fig. 12.

\section{DISCUSSION}

As the numerical examples demonstrate, PINN with the adaptive $\sin (x)$ áctivation function solves the Helmholtz equations for isotropic and anisotropic media. The network accepts the spatial coordinate values as the input data, and outputs the rea $\mathrm{and}$ imaginary parts of the wavefield solution. The loss function used to train the network is given by the proper Helmholtz equation, which controls the wave propagation. Parameters affecting the wave shape like frequency, source location, velocity, and anisotropie parameters are given as non-trainable parameters in the loss function. In the previous work, the developed PINN-based Helmholtz equation solver for the scattered wavefields had three unresolved limitations (Alkhalifah et al. 2020a,b; Song et al. 2021): 1. the resulting scattered wavefields had low resolution, and the scattering components in the wavefields were absent; 2 . it relied on obtaining analytical so-

(a)

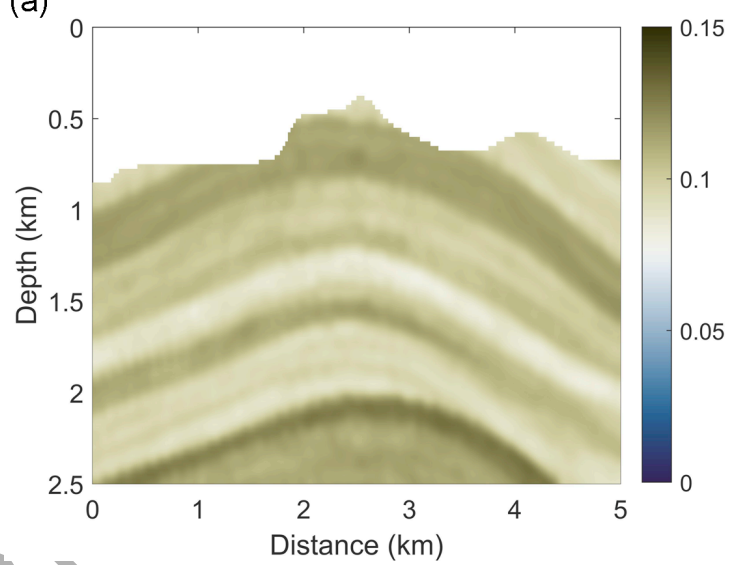

(b)

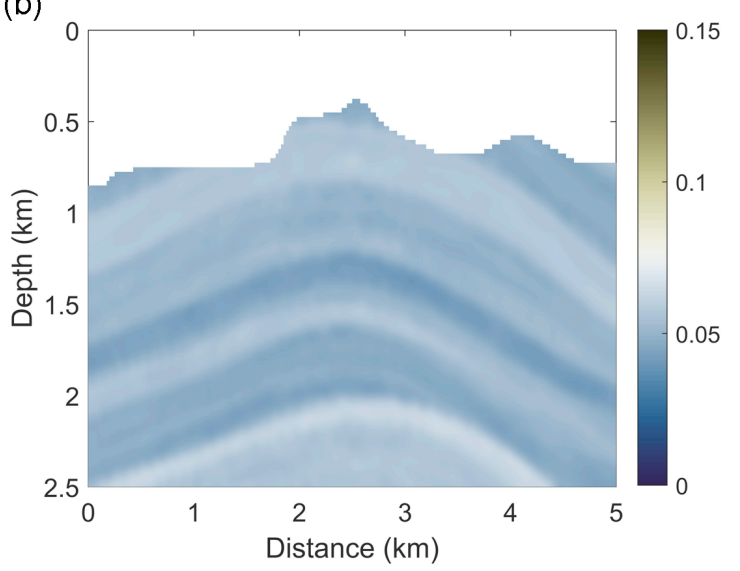

Figure 14. The true (a) $\epsilon$ and (b) $\delta$ models with irregular topography. 
(a)

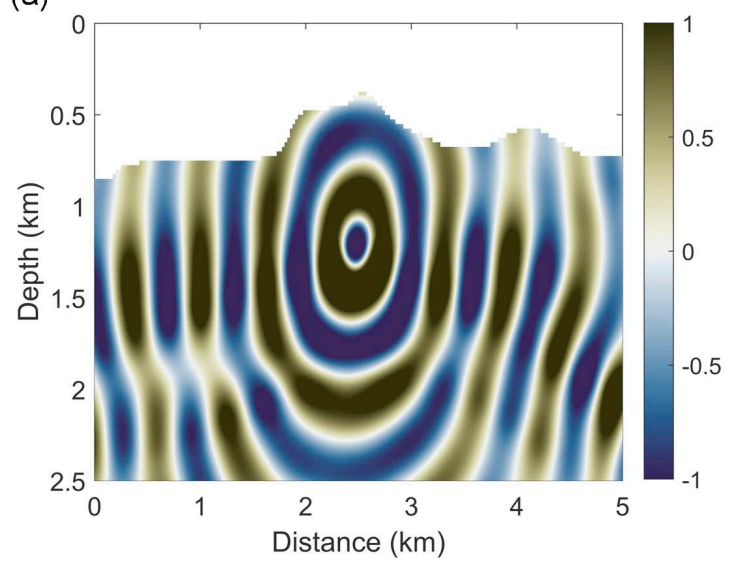

(b)

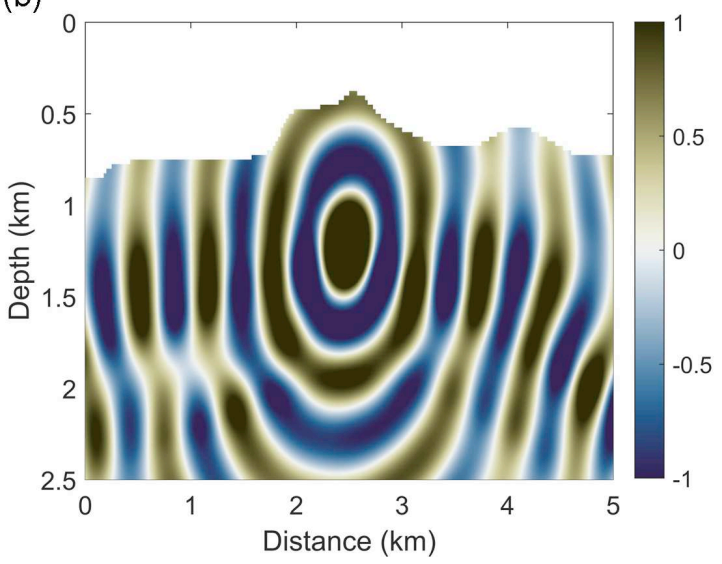

Figure 15. The (a) real and (b) imaginary parts of the PINN-predicted pressure wavefield solution with adaptive $\sin (x)$ activation function for the anisotropic topography model of $5 \mathrm{~Hz}$.

lutions for the background wavefields. However, analytical solutions are only available to a limited type of wave equations with specific source functions, like a point source. 3. it failed to produce the wavefields for large-contrast media. In this paper, we resolve these issues using the proposed implementation. As a result, the proposed method is a yersatile framework to solve wave equations for any media with high resolution and reasonáble accuracy.

In all our experiments we used regular grid points as our training data. The reason is that we want to make sure the source is well sampled. Otherwise, the sparsity nature of the source will cause convergence issues, which will lead to divergence for the optimization. Using random locations within the model domain as training data will generate equally accurate predictions if part of these random locations reasonably sample the source area. In this case, the velocity model and the source function need to be evaluated through interpolation at these random locations. Predicting the wavefield on a regular grid can be used to test the network and decide whether overfitting is encountered. However, our relatively small NNs used to represent the wavefield often alleviate this problem.

Using PINN method to solve for the frequency-domain wavefield, we need to adjust the network and the training epoch number according to the size and complexity of the model, or more precisely, the complexity of the wavefield. For a large and complex model, we need more neurons and hidden layers to represent the the resulting complex wavefield. As a result, more epochs are required to achieve convergence in the training process. Frequency is another important factor controling the complexity of the wavefield. As the frequency increase, the sinusoidal nature of the resulting frequency-domain wavefield becomes more obvious, and the wavefield function becomes more complex. Thus, for the same model, more training points 
and epochs are necessary. In addition, as we are using an adaptive sine activation function, the adaptive parameter will try to match the frequency. Thus, higher frequencies will require a larger $w_{0}$, to match the expected wavenumber range. However, large variations in the model velocity will complicate the adaptation process.

The PML boundary condition is used in our proposed method, even though it requires an extension of the model, which will increase the training cost. The frequency-domain wavefields should contain both real and imaginary parts. If no boundary conditions are applied, the resulting wavefield solution will have only the real part for a delta function source. In this paper, we do not consider the free surface boundary condition. If the free-surface boundary condition is applied, we include an additional term to the loss function that forces the stress or pressure to zero. Otherwise, PINN through its optimization implementation do not admit reflections along boundaries.

For anisotropic media, we use a coupled system of second-order partial differential equations to simulate the acoustic wave propagation. To generate wavefield solutions considering anisotropy using PINNs, we incorporate both equations of the coupled system in the loss function. As the loss function for anisotropic media includes more terms than the isotropic case, it requires more training epochs to converge. Besides the pressure wavefield $p$, the auxiliary perturbation wavefield $q$ is also used as an output parameter

Compared to the commonly used activation function $\tanh (x)$, which is also originally used in PINN (Raissi et al. 2019a), the $\sin (x)$ activation function is found to be more stable (Raissi et al. 2019b). Sitzmann et al. (2020) state that it is important to initialize the weights in the first layer of the sine network with a fixed coefficient. To avoid the extra cost of trial and error tests in finding an optimal coefficient, we use an adaptive $\sin (x)$ activation function, which optimizes the coefficient in the training process to achieve the best training performance.

The main limitation of the proposed method is that we need to train a new network for each individual model, and also for each individual source and frequency. In seismic imaging and waveform inversionapplications, a number of sources are required to illuminate the subsurface structures. Thus, it will be costly to use the proposed method to generate wavefields for many sources. However, we can introduce source location as extra inputs to the network to generate wavefield solutions for multiple sources using just one single network (Alkhalifah et al. 2020a). Additionally, this new network can be used to invert for a high-resolution velocity model (Song \& Alkhalifah 2020b).

Currently, the training cost of PINN in solving the 2D Helmholtz equation is higher than the numerical method, which could change as we further improve the PINN functionality. 
However, the main advantage of PINN is its versatility in handling various media and model with irregular shapes. By comparison, significant modifications are required to convert numerical Helmholtz solvers to work on anisotropic media, as an example. For PINN, we only need to insert the anisotropic wave equation in the loss function. Additionally, the proposed method can be a stepping stone for future applications. When the Helmholtz equation represents more complex physics like orthorhombic elasticity, fluid-saturation porosity, in 3D media, the computational cost of the numerical method will increase dramatically, and in some cases such solutions are not available (Yang et al. 2020; Yang \& Malcolm 2021). In this case, the proposed method will be more computationally attractive. We will show this feature in a follow-up paper. As for different models, transfer learning is a good tool to accelerate the training process (Pan \& Yang 2009; Waheed et al. 2021), which means that we can use a trained network from a previous model to the next model to improve the training efficiency.

Besides PINN, there are other machine learning based methods to solve PDEs, and most of them are data-driven. For example, convolutional NN (CNN) was used to perform fast fluid flow simulation corresponding to inviscid Euler Equations (Tompson et al. 2017), and to resolve Reynolds-averaged NavierStokes (RANS) equations (Thuerey et al. 2020). (Moseley et al. 2020) used an autoencoder to achieve fast seismic data símulation. Neural operator is a different concept from CNN, which can be used to learn the mapping between function spaces (Li et al. 2020b). A Fourier space domain implementation of the neural operator, which is referred to as Fourier neural operator (FNO), can accelerate the training process ( $\mathrm{Li}$ et al. 2020a; Konuk \& Shragge 2021). However, most of these methods are devoted to representing the PDE operator, not developing a wavefield solution, and the cost of their training can be expensive.

\section{CONCLUSIONS}

We applied the phyśsics-informed neural networks (PINNs) to solve the Helmholtz equation for isotropic and anisotropic media. We use a fully-connected neural network accepting the spatial coordinates of points in the solution space as input data to predict the real and imaginary parts of the pressure wavefield. Using an adaptive $\sin (x)$ activation function to connect the outputs between hidden layers, we can achieve fast convergence in the training of the network, resulting in high-resolution wavefield solutions. The proposed method has resilience and versatility in predicting frequency-domain wavefields for different media and model shapes. The trained network becomes a function of spatial coordinate values, and it 


\section{Song et al.}

has no limitations on the shape of the solution space. Applications on isotropic and anisotropic models show that the proposed method can provide reasonably accurate wavefield solutions.

\section{ACKNOWLEDGMENT}

We thank KAUST for its support and the SWAG group for the collaborative environment. This work utilized the resources of the Supercomputing Laboratory at King Abdullah University of Science and Technology (KAUST) in Thuwal, Saudi Arabia, and we are grateful for that. We thank the editor, Dr Andrew Valentine, assistant editor, Louise Alexander, and Dr. Martijn van den Ende and one anonymous reviewer, for their critical and helpful review of the manuscript. We thank Dr. Fabio Crameri for releasing a perceptually-uniform color map.

\section{DATA AVAILABILITY}

There are no new data associated with this article. The codes are available at https://github.com/songc0a/PINN-Helmholtz-solver-adaptive-sine to allow other researchers to reproduce the examples and evaluate the proposed method. 


\section{REFERENCES}

Abadi, M., Agarwal, A., Barham, P., Brevdo, E., Chen, Z., Citro, C., Corrado, G. S., Davis, A., Dean, J., Devin, M., et al., 2015. TensorFlow: Large-scale machine learning on heterogeneous systems.

Alkhalifah, T., 1998. Acoustic approximations for processing in transversely isotropic media, Geophysics, 63(2), 623-631.

Alkhalifah, T., 2000. An acoustic wave equation for anisotropic media, Geophysics, 65(4), 1239-1250.

Alkhalifah, T., Song, C., \& Waheed, U. b., 2020a. Machine learned Green's functions that approximately satisfy the wave equation, in SEG International Exposition and 90th Annual Meeting, vol. 2020, pp. 2638-2642, Society of Exploration Geophysicists.

Alkhalifah, T., Song, C., Waheed, U. b., \& Hao, Q., 2020b. Wavefield solutions from machine learned functions that approximately satisfy the wave equation, in 82nd EAGE Annual Conference \& Exhibition, vol. 2020, pp. 1-5, European Association of Geoscientists \& Engineers.

Baydin, A. G., Pearlmutter, B. A., Radul, A. A., \& Siskind, J. M., 2017. Automatic differentiation in machine learning: a survey, The Journal of Machine Learning Research, 18(1), 5595-5637.

Berenger, J.-P. et al., 1994. A perfectly matched layer for the absorption of electromagnetic waves, Journal of computational physics, 114(2), 185-200.

Bishop, C. M., 2006. Pattern recognition and machine learning, springer.

Calderón-Mac ı'as, C., Sen, M. K., \& Stoffa, P. L., 1998. Automátic umo correction and velocity estimation by a feedforward neural network, Geophysics, 63(5), 169611707 .

Chen, Z., Cheng, D., Feng, W., \& Wu, T., 2013. An optimal 9-point finite difference scheme for the Helmholtz equation with PML, International Journat of Numerical Analysis 83 Modeling, 10(2), $389-410$.

Clayton, R. \& Engquist, B., 1977. Absorbing boundary conditions for acoustic and elastic wave equations, Bulletin of the seismological society of America, 67(6), 1529-1540.

Dai, H. \& MacBeth, C., 1995. Automatic picking of seismic arrivals in local earthquake data using an artificial neural network, Geophysical journal international, 120(3), 758-774.

Duveneck, E. \& Bakker, P. M. 2011. Stable p-wave modeling for reverse-time migration in tilted TI media, Geophysics, 76(2), S65-S75.

Duveneck, E., Milcik, P., Bakker, P. M., \& Perkins, C., 2008. Acoustic VTI wave equations and their application for anísotropic reverse-time migration, in SEG Technical Program Expanded Abstracts 2008, pp. 2186 2190, Society of Exploration Geophysicists.

Gentili, S. \& Michelini, A., 2006. Automatic picking of p and s phases using a neural tree, Journal of Seismology, 10(1), 39-63.

Glorot, X. \& Bengio, Y., 2010. Understanding the difficulty of training deep feedforward neural networks, in Proceedings of the thirteenth international conference on artificial intelligence and statisties, pp. 249-256, JMLR Workshop and Conference Proceedings.

Hastings, F. D., Schneider, J. B., \& Broschat, S. L., 1996. Application of the perfectly matched layer 
(PML) absorbing boundary condition to elastic wave propagation, The Journal of the Acoustical Society of America, 100(5), 3061-3069.

Higdon, R. L., 1992. Absorbing boundary conditions for acoustic and elastic waves in stratified media, Journal of Computational Physics, 101(2), 386-418.

Hornik, K., Stinchcombe, M., \& White, H., 1989. Multilayer feedforward networks are universal approximators, Neural networks, 2(5), 359-366.

Jo, C.-H., Shin, C., \& Suh, J. H., 1996. An optimal 9-point, finite-difference, frequency-space, 2-D scalar wave extrapolator, Geophysics, 61(2), 529-537.

Karimpouli, S. \& Tahmasebi, P., 2020. Physics informed machine learning: Seismic wave equation, Geoscience Frontiers, 11(6), 1993-2001.

Kaur, H., Fomel, S., \& Pham, N., 2020. Seismic ground-roll noise attenuation using deep learning, Geophysical Prospecting, 68(7), 2064-2077.

Konuk, T. \& Shragge, J., 2021. Physics-guided deep learning using fourier neural operator for solving the acoustic vti wave equation, in 82nd EAGE Annual Conference \& Exhibition, vol. 2021, pp. 1-5, European Association of Geoscientists \& Engineers.

Leshno, M., Lin, V. Y., Pinkus, A., \& Schocken, S., 1993. Multilayer feedforward networks with a nonpolynomial activation function can approximate any function, Neural hetworks, 6(6), 861-867.

Li, Z., Kovachki, N., Azizzadenesheli, K., Liu, B., Bhattacharya, K., Straart, A., \& Anandkumar, A., 2020a. Fourier neural operator for parametric partial differential equations, arXiv preprint arXiv:2010.08895.

Li, Z., Kovachki, N., Azizzadenesheli, K., Liu, B., Bhattacharya, K., Stuart, A., \& Anandkumar, A., 2020b. Neural operator: Graph kernel network for partial differential equations, arXiv preprint arXiv:2003.03485.

Liu, D. C. \& Nocedal, J., 1989. On the limited memory BFGS method for large scale optimization, Mathematical programming, 45(1-3), 503-528.

Marfurt, K. J., 1984. Accuracy of finite-difference and finite-element modeling of the scalar and elastic wave equations, Geophysics, $49(\widehat{5}), 533-549$.

Moseley, B., Nissen-Meyer, ₹ \& Markham, A., 2020. Deep learning for fast simulation of seismic waves in complex media, Solid Earth, 11(4), 1527-1549.

Operto, S., Virieux, JJ., Ribodetti, A., \& Anderson, J. E., 2009. Finite-difference frequency-domain modeling of viscoacoustic wave propagation in 2D tilted transversely isotropic (TTI) media 2D acoustic wate modeling in TTI media, Geophysics, 74(5), T75-T95.

Pan, S. J. \& Yang, Q., 2009. A survey on transfer learning, IEEE Transactions on knowledge and data engineéring, 22(10), 1345-1359.

Postma. G., 1955. Wave propagation in a stratified medium, Geophysics, 20(4), 780-806.

Pratt, R. G., 1990. Frequency-domain elastic wave modeling by finite differences; a tool for crosshole seismic imaging, Geophysics, 55(5), 626-632. 
Pratt, R. G., 1999. Seismic waveform inversion in the frequency domain; Part 1, Theory and verification in a physical scale model, Geophysics, 64(3), 888-901.

Raissi, M., Perdikaris, P., \& Karniadakis, G. E., 2019a. Physics-informed neural networks: A deep learning framework for solving forward and inverse problems involving nonlinear partial differential equations, Journal of Computational Physics, 378, 686-707.

Raissi, M., Wang, Z., Triantafyllou, M. S., \& Karniadakis, G. E., 2019b. Deep learning of vortexinduced vibrations, Journal of Fluid Mechanics, 861, 119-137.

Shukla, K., Di Leoni, P. C., Blackshire, J., Sparkman, D., \& Karniadakis, G. E., 2020. Physicsinformed neural network for ultrasound nondestructive quantification of surface breaking cracks, arXiv preprint arXiv:2005.03596.

Sibi, P., Jones, S. A., \& Siddarth, P., 2013. Analysis of different activation functions using back propagation neural networks, Journal of theoretical and applied information technology, 47(3), 1264 1268.

Sitzmann, V., Martel, J., Bergman, A., Lindell, D., \& Wetzstein, G., 2020. Implicit neural representations with periodic activation functions, Advances in Neural Information Processing Systems, 33, 7462-7473.

Smith, J. D., Azizzadenesheli, K., \& Ross, Z. E., 2020. Eikonet: Solving the eikonal equation with deep neural networks, IEEE Transactions on Geoscience and Remote Sensing.

Song, C. \& Alkhalifah, T., 2020a. An efficient wavefield inversion for transversely isotropic media with a vertical axis of symmetry, Geophysics, 85(3), R195-R206.

Song, C. \& Alkhalifah, T., 2020b. Wavefield reconstruction inversion via machine learned functions, in SEG Technical Program Expanded Abstracts 2020, pp. 1710-1714, Society of Exploration Geophysicists.

Song, C., Alkhalifah, T., \& Waheed, U. B., 2021. Solving the frequency-domain acoustic VTI wave equation using physics-informed neural networks, Geophysical Journal International, 225, 846-859. Thomsen, L., 1986. Weak elastic anisotropy, Geophysics, 51(10), 1954-1966.

Thuerey, N., Weißenow, K., Prantl, L. \& Hu, X., 2020. Deep learning methods for reynolds-averaged navier-stokes simulations of airfoil flows, AIAA Journal, 58(1), 25-36.

Tompson, J., Schlachter, K., Sprechmann, P., \& Perlin, K., 2017. Accelerating eulerian fluid simulation with convolutional/networks, in International Conference on Machine Learning, pp. 3424-3433, PMLR.

Tsynkov, S. \& Turkel, E., 2001. 6.1 a cartesian perfectly matched layer for the helmholtz equation, Absorbing Boundaries and Layers, Domain Decomposition Methods: Applications to Large Scale Computers, p. 279.

Uhrig, L. F. \& Van Melle, F. A., 1955. Velocity anisotropy in stratified media, Geophysics, 20(4), 774- 779 .

Valentine, A. P. \& Trampert, J., 2012. Data space reduction, quality assessment and searching of 
seismograms: autoencoder networks for waveform data, Geophysical Journal International, 189(2), 1183-1202.

van den Ende, M. P. \& Ampuero, J.-P., 2020. Automated seismic source characterization using deep graph neural networks, Geophysical Research Letters, 47(17), e2020GL088690.

Van der Baan, M. \& Jutten, C., 2000. Neural networks in geophysical applications, Geophysics, 65(4), 1032-1047.

Waheed, U., Haghighat, E., Alkhalifah, T., Song, C., \& Hao, Q., 2020a. Eikonal solution using physics-informed neural networks, in 82nd EAGE Annual Conference 63 Exhibition, vol. 2020, pp. 1-5, European Association of Geoscientists \& Engineers.

Waheed, U. b. \& Alkhalifah, T., 2015. An efficient wave extrapolation method for anisotropic media with tilt, Geophysical Prospecting, 63(5), 1126-1141.

Waheed, U. b., Haghighat, E., \& Alkhalifah, T., 2020b. Anisotropic eikonal solution using physicsinformed neural networks, in SEG Technical Program Expanded Abstracts 2020, pp. 1566-1570, Society of Exploration Geophysicists.

Waheed, U. b., Haghighat, E., Alkhalifah, T., Song, C., \& Hao, Q., 2021. PINNeik: Eikonal solution using physics-informed neural networks, Computers $\mathscr{E}$ Geosciences, p. 104833

Wang, H. \& Alkhalifah, T., 2021. Direct microseismic event location and characterization from passive seismic data using convolutional neural networks, Geophysics, 86(6), KS109-KS121.

Wang, H., Alkhalifah, T., bin Waheed, U., \& Birnie, C., 2021a. Data-driven microseismic event localization: an application to the oklahoma arkoma basin hydraulic fracturing data, IEEE Transactions on Geoscience and Remote Sensing.

Wang, H., Liu, Y., Yin, C., Zhao, P., \& Cao, J., 2021b Physics-informed deep learning for magnetotelluric 2d forward modeling, in 82nd EAGE Annual Conference 83 Exhibition, vol. 2021, pp. 1-5, European Association of Geoscientists \& Engineers.

Wang, S., Wang, H., \& Perdikaris, P., 2021c. On the eigenvector bias of fourier feature networks: From regression to solving multi-scale pdes with physics-informed neural networks, Computer Methods in Applied Mechanics and Engineering, 384, 113938.

Yang, Q. \& Malcolm, A., 2021. Frequency domain full-waveform inversion in a fluid-saturated poroelastic medium, Geophysical Journal International, 225(1), 68-84.

Yang, Q., Zhou, B, RRiahi, M. K., \& Al-Khaleel, M., 2020. A new generalized stiffness reduction method for $2 \mathrm{~d} / 2.5 \mathrm{~d}$ frequency-domain seismic wave modeling in viscoelastic anisotropic media, Geophysics, 85(6), T315-T329.

Zhou, H., Zhang, G., \& Bloor, R., 2006a. An anisotropic acoustic wave equation for VTI media, in 68th EAGE Conference and Exhibition incorporating SPE EUROPEC 2006, pp. cp-2, European Association of Geoscientists \& Engineers.

Zhou, H., Zhang, G., \& Bloor, R., 2006b. An anisotropic acoustic wave equation for modeling and migration in 2D TTI media, in SEG Technical Program Expanded Abstracts 2006, pp. 194-198, 
Society of Exploration Geophysicists.

Zhu, W. \& Beroza, G. C., 2019. PhaseNet: a deep-neural-network-based seismic arrival-time picking method, Geophysical Journal International, 216(1), 261-273. 